\title{
Biological therapies in the treatment of inflammatory disease and cancer: impact on pulmonary infection
}

\author{
Ricardo J. José ${ }^{1}$, Maria Mouyis ${ }^{2}$ \\ ${ }^{1}$ Centre for Inflammation and Tissue Repair, UCL Respiratory, University College London, London, UK; ${ }^{2}$ Department of Rheumatology, \\ Northwick Park Hospital, Harrow, UK \\ Contributions: (I) Conception and design: All authors; (II) Administrative support: None; (III) Provision of study materials or patients: None; (IV) \\ Collection and assembly of data: None; (V) Data analysis and interpretation: None; (VI) Manuscript writing: All authors; (VII) Final approval of \\ manuscript: All authors. \\ Correspondence to: Dr. Maria Mouyis. Department of Rheumatology, Northwick Park Hospital, Watford Road, Harrow, HA1 3 UJ, UK. \\ Email: m.mouyis@nhs.net.
}

\begin{abstract}
Biological therapies are increasingly used for the treatment of inflammatory conditions in the realms of rheumatology, dermatology, and gastroenterology due to their ability to target specific cytokines in the inflammatory cascade. The impact of these biologic therapies is immunosuppression leading to an increased risk of infection. This review focuses on commonly used biologic agents in the treatment of inflammatory conditions and cancer and their impact on pulmonary infections. We have summarized potential pathogens in this group of patients. The hope being that this will increase awareness and therefore prevention timely diagnosis and successful treatment of patients receiving biologic therapies. It is also important to note that it is not solely the choice of an agent that predisposes to particular infections. Concomitant factors that might increase an individuals' risk of contracting an infection include the underlying disease, comorbid diseases, increased age, and other medical treatment as well as exposure to opportunistic pathogens. In the treatment of cancers many immunotherapies are being developed. The most notable adverse effects from immunotherapy are due to stimulation of the immune response, and these may mimic infection by causing flu-like symptoms and breathlessness due to pneumonitis. The treatment of this is immunosuppression, further leading to an increased risk of infection. Biologic therapies have been a revolution in the treatment of inflammatory conditions and cancers. They have improved outcomes and quality of life for patients. However, the use of these drugs needs to be balanced against the risk of infection and every patient needs to be assessed on an individual basis.
\end{abstract}

Keywords: Biologics; respiratory infection; pneumonia; immunotherapy; cancer; tuberculosis

Received: 02 August 2017; Accepted: 04 September 2017; Published: 13 September 2017.

doi: 10.21037/arh.2017.09.03

View this article at: http://dx.doi.org/10.21037/arh.2017.09.03

\section{Introduction}

Biological therapies are increasingly used for the treatment of inflammatory conditions in the realms of rheumatology, dermatology, and gastroenterology due to their ability to target specific cytokines in the inflammatory cascade. More recently these compounds have emerged in oncology to target cancer cells directly; stimulate the body's response against the cancer cells, or inhibit pathways that promote tumour growth. These agents have led to dramatic improvements in treating disease, prolonging life and improving quality of life. However, targeting the immune system with these drugs increases the risk of respiratory infections, which are an important cause of morbidity and mortality in these patients (1). The spectrum of potential pathogens known to cause respiratory infections in these individuals has increased, and to identify the subset of pathogens that may be the culprits of the infection it is 
Table 1 Potential respiratory pathogens according to immune defect

\begin{tabular}{ll}
\hline Immune defect & Potential respiratory pathogens \\
\hline neutropenia/impaired & Gram positive bacteria (e.g., Staphylococcus aureus, Streptococcus pneumoniae, Nocardia species) \\
& Gram negative bacteria (e.g., Klebsiella pneumoniae) \\
& Fungi (e.g., Aspergillus species, Candida species) \\
T-cell mediated immunity & Herpesviruses (e.g., herpes simplex virus, Cytomegalovirus) \\
& Respiratory viruses (e.g., influenza) \\
& Fungi (e.g., Pneumocystis jirovecii, Histoplasma capsulatum, Cryptococcus neoformans) \\
& Mycobacteria \\
& Nocardia species \\
Legionella pneumophila & Encapsulated bacteria (e.g., Streptococcus pneumoniae, Haemophilus influenzae) \\
\hline
\end{tabular}

important to know the nature of the underlying immune defect. This is of paramount importance for prevention, timely diagnosis and successful treatment of patients receiving biological therapies (Table 1). It is also important to note that it is not solely the choice of an agent that predisposes to particular infections. Concomitant factors that might increase an individuals' risk of contracting an infection include the underlying disease, comorbid diseases (e.g., diabetes mellitus), increased age, and other medical treatment (e.g., use of high dose corticosteroids). Exposure to opportunistic pathogens is also increased by living or travelling in endemic areas, or through occupational exposure, contaminated food, soil and water $(2,3)$. Here we provide a concise review of established biological therapies and their impact on respiratory infection. The investigations required for the detection of opportunistic respiratory pathogens and their treatment are not discussed here as they are discussed in detail elsewhere $(2,4)$.

\section{Biological therapy in autoimmune and inflammatory diseases}

\section{Tumour necrosis factor (TNF) inbibitors}

TNF is a pleiotropic pro-inflammatory cytokine central to many aspects of the immune response during disease and in the host's response to infection. When present in excessive concentrations it is responsible for the destructive inflammatory processes that result in bystander tissue damage and consequently is a target in the treatment of various inflammatory diseases. The pharmacological class of
TNF inhibitors includes: (I) etanercept, a soluble p75 TNF receptor fusion protein; (II) infliximab, a chimeric antiTNF antibody; (III) adalimumab, a fully human monoclonal anti-TNF antibody; (IV) certolizumab, an antigen-binding fragment (Fab') of a humanized monoclonal antibody coupled to polyethylene glycol; and (V) golimumab, human anti-TNF-alpha monoclonal antibody.

TNF stimulates macrophages to produce cytotoxic metabolites, thereby increasing phagocytic killing activity. Consequently, inhibition of TNF impairs macrophage phagocytosis that predisposes to infection. Although the mechanism is not clear anti-TNF therapy also results in significant neutropenia (5). Overall, this results in the inability to eliminate pathogens and increases the risk of developing serious infections requiring hospitalisation, which is seen with the use of all TNF inhibitors. However, it is important to note that in a meta-analysis including $>50,000$ participants conducted in 2011 a statistically significant increased risk was demonstrated only for certolizumab [odds ratio (OR) 3.51, 95\% CI: 1.59-7.79] (6). More recently, a 2015 meta-analysis of patients with rheumatoid arthritis, who received biologic drugs, found that all the classes of TNF inhibitors increased the risk of serious infections compared to traditional disease-modifying anti-rheumatic drugs (DMARD) (6). The risk was higher when high doses were used compared to standard doses (OR 1.90, 95\% CI: $1.50-2.39$ vs. OR 1.31, 95\% CI: $1.09-$ 1.58) (7), suggesting that the risk of infection may be dose dependent. Other risk factors which are demonstrated to increase the possibility of infection in patients receiving TNF inhibitors include age $\geq 65$ years, concomitant use of 
immunosuppressants and co-morbidities such as chronic obstructive pulmonary disease (8). Furthermore, the risk of infection is deemed highest when starting the TNF inhibitor (9). The British Society for Rheumatology Biologics Register reported that the risk of infection is 4.6 (95\% CI: 1.8-11.9) times greater in the first 90 days (10), highlighting that during this initial period of therapy caution is required in patients developing symptoms and signs of infection.

In the first randomised controlled trial of Infliximab, one patient developed tuberculosis and another coccidioidomycosis (11). Since then there has been increased recognition of the association between the use of TNF inhibitors and the development of active infection, especially with opportunistic pathogens (3). Notably, due to loss of cellular and humoral immunity with anti-TNF therapy, individuals are also predisposed to infections with pathogens that normally cause infection in healthy individuals such as the respiratory viruses and Streptococcus pneumoniae, and in these cases, the ongoing use of TNF inhibitors can result in invasive infection. Likewise, TNF inhibitors increase the risk of infection with Legionella pneumophila, with a relative risk of Legionnaire's disease in rheumatoid arthritis patients treated with anti-TNF inhibitors estimated to be between 17 to 21 in comparison with the overall risk in France (12).

Of the respiratory infections associated with anti-TNF therapy infection with Mycobacterium tuberculosis is the most notable, with a 25 -fold increased risk of reactivating latent M. tuberculosis following initiation of treatment (13). The risk is increased because TNF together with IFN- $\gamma$ plays a major role in elimination of mycobacteria and confines mycobacteria to granulomas, which keeps the disease in a latent state (14). The risk of reactivation is greater with the use of infliximab and adalimumab compared to etanercept $(15,16)$. Furthermore, anti-TNF therapy increases the risk of non-tuberculous mycobacterial infections, including $M$. avium complex, $M$. chelonae, $M$. marinum and $M$. abscessus (14). Therefore, current recommendation is to screen individuals for active and latent $M$. tuberculosis infection by taking a detailed history, performing a physical examination, obtaining an interferon gamma release assay (IGRA) and/or tuberculin skin test (TST), and a chest radiograph in those with a positive TST/IGRA or if symptoms suggest active disease. In most cases, an IGRA is sufficient for screening, but in individuals with significant risk factors for previous tuberculosis exposure, the TST should also be carried out if the initial IGRA is negative. If latent tuberculosis infection is diagnosed then treatment should be commenced before initiating anti-TNF therapy (17).

TNF also plays a key role in the containment of other granulomatous infections including fungal infections, particularly in endemic areas, where fungal infections are a recognised complication of treatment with TNF inhibitors, particularly with infliximab $(18,19)$. Of concern is that many cases of pulmonary and disseminated histoplasmosis, coccidioidomycosis and blastomycosis are missed in these individuals and treatment initiation is delayed, which can result in poor outcomes (20). A high index of suspicion is required in the presence of dyspnoea, fever, and malaise, as well as radiological features of interstitial pneumonitis, mediastinal granulomatous lymphadenitis and mediastinal fibrosing mediastinitis (21). In endemic areas for coccidioidomycosis, despite most infections occurring de novo, it is best to perform a chest radiograph and coccidioidal serologic tests prior to the initiation of TNF inhibitors as re-activation of latent infection can occur (19). Due to neutropenia associated with TNF inhibitors, infections with Aspergillus species (e.g., A. fumigatus), which are ubiquitous environmental fungi, are also of concern particularly in the presence of other immunosuppressants (22). Pneumocystis jirovecii pneumonia is also reported in individuals receiving infliximab, especially within the first month of receiving the infusion (23) and when used concomitantly with high doses of glucocorticoids (24). Consequently, patients receiving both high dose corticosteroids and TNF-inhibitor should be considered for PCP prophylaxis.

TNF also plays a key role in the immune response against viral infections, and respiratory infections with respiratory viruses (respiratory syncytial virus, parainfluenza, influenza, adenovirus, metapneumovirus, coronavirus and rhinovirus) are relatively common in individuals treated with TNF-inhibitors. Re-activation of latent infections with viruses from the Herpesviridae family (e.g., human herpes virus, cytomegalovirus, and Epstein-Barr virus) are not frequently seen, but a high index of suspicion for these is required. In immunocompromised individuals, these viral infections can be severe and fatal if not identified early so that TNF inhibitor therapy can be withheld and anti-viral treatment commenced $(3,25)$.

\section{Rituximab}

Rituximab is a chimeric/humanised monoclonal antibody that acts on $\mathrm{CD}_{20}{ }^{+}$cells and leads to $\mathrm{B}$ cell depletion via apoptosis and complement activation before these cells 
develop into plasma cells (26-28). In addition to B-cell depletion, rituximab results in hypogammaglobulinemia, which predisposes to recurrent respiratory infection and may require treatment with intravenous immunoglobulin replacement therapy (29). It is, therefore, crucial to obtain baseline serum immunoglobulin levels (IgG, IgA, and $\operatorname{IgM}$ ) prior to initiation of rituximab therapy and periodic monitoring to identify persistent immunoglobulin deficiencies before the onset of severe infections. In a large study of rheumatoid arthritis patients that received rituximab, serious infections were encountered in $7 \%$ of individuals with most having pneumonia, especially when low IgM levels were encountered (30). With the immunoglobulin deficiency, patients receiving rituximab will not mount adequate responses to vaccines, particularly polysaccharide vaccines such as that against $S$. pneumoniae. Therefore, to prevent against pneumococcal pneumonia in this high-risk group the pneumococcal vaccine should be administered before initiation of rituximab treatment.

Unlike with TNF inhibitors there is no need to screen individuals for $M$. tuberculosis infection, but it is important to bear in mind that the risk of re-activation of latent tuberculosis infection is heightened when used concomitantly with other immunosuppressive agents such as high dose corticosteroids (31).

\section{Belimumab}

B-lymphocyte stimulator (BLyS) is a transmembrane protein which is a B-cell activating factor and therefore promotes maturation of $\mathrm{B}$-cells into plasma cells and the production of antibodies (32). Belimumab is a human monoclonal antibody that binds to soluble human B-lymphocyte stimulator protein (BLyss) to inhibit its biologic activity in patients with systemic lupus erythematosus (SLE) (33). It increases the development of serious respiratory infections during the first year of treatment, with particular reports of coccidioidomycosis and cytomegalovirus pneumonia. However, in a randomised clinical trial patients treated with belimumab did not have rates of serious infection greater than those treated with placebo (34).

\section{Tocilizumab}

Tocilizumab is a humanised monoclonal antibody targeting the interleukin (IL)-6 receptor and blocks the downstream signalling effects of IL-6 on the function of neutrophils, T cells, B cells, and monocytes. Additionally, IL-6 is a potent inducer of the hepatic acute phase response and secretion of c-reactive protein and is an endogenous pyrogenic (35). Therefore, inhibition of IL-6 may predispose to the development of severe infection in the absence of a febrile and pro-inflammatory response, which may lead to diagnostic and therapeutic delays. Tocilizumab can lead to neutropenia, but the adverse effect is usually transient. However, there remains an increased risk of respiratory infection comparable to that seen with TNF inhibitors and there is a requirement to screen for latent mycobacterium tuberculosis infection prior to initiation of treatment (36). The risk is particularly increased in those $>65$ years of age, with underlying co-morbid respiratory disease, on $>5 \mathrm{mg} /$ day corticosteroids, or on a concomitant DMARD (34-37).

\section{Abatacept}

Abatacept is a soluble fusion protein of human IgG1 to CTLA4, which is a T-cell surface receptor. The CTLA4 binds to the $\mathrm{B}$ cell thus preventing $\mathrm{B}$ cell activation, and $\mathrm{T}$ cell mediated cytokine release (38). Respiratory infections with opportunistic pathogens were reported with abatacept therapy (9) but overall the available data do not suggest a significantly increased risk of serious infections compared to placebo. The risk for reactivation of $M$. tuberculosis associated with abatacept therapy is currently unclear, but the manufacturer recommends screening for latent infection before treatment initiation $(9,32)$.

\section{Tofacitinib}

Tofacitinib is a Janus kinase (JAK) inhibitor that influences multiple downstream signalling pathways. The risk of infections with tofacitinib is similar to that of DMARDs. However, the rate of serious infections with tofacitinib is $3 / 100$ patient years and increases in advanced age, those with diabetes mellitus and with concomitant use of corticosteroids (39). The greatest risk with tofacitinib appears to be with infection with herpes zoster and vaccination is recommended prior to initiation of treatment (40).

\section{Eculizumab}

Eculizumab is a humanized monoclonal antibody that binds to the $\mathrm{C} 5$ component of complement, inhibiting terminal complement activation $(32,41)$. Eculizumab increases the risk of life-threatening neisserial infections, including $N$. meningitidis and the manufacturers prescribing 
information includes a boxed warning describing the risk, the need for meningococcal vaccination at least two weeks prior to treatment, and the importance of monitoring for meningococcal infection (42). Since Eculizumab inhibits complement activation it will also increase the risk of respiratory infection with other encapsulated pathogens such as $S$. pneumoniae and $H$. influenzae (43).

\section{Anakinra}

The IL-1 receptor antagonist (IL-1Ra) is a naturally occurring glycoprotein inhibitor of IL-1 by binding to the IL-1 receptor and anakinra is the recombinant human IL$1 \mathrm{Ra}$ that functions through competitive binding to the IL-1 receptor (44). The risk of serious infections with anakinra is significantly increased compared to placebo with an OR 4.05 (95\% CI: 1.22-16.8) (6). This increases with combination etanercept, and therefore the use of anakinra in combination with other biological therapies is not recommended (45). Regarding the reactivation of latent $M$. tuberculosis there is one case report in the literature (46) and overall the data does not suggest that anakinra significantly increases the risk of developing tuberculosis (6).

\section{Ustekinumab}

Ustekinumab is a human IgG1 kappa monoclonal antibody that binds to the shared p40 subunit of IL-12 and IL23 , preventing the binding of the pro-inflammatory cytokines IL-12 and IL-23 to their cell surface receptor. It results primarily in impairment of natural killer (NK) cell activation, as well as CD4+ T-cell differentiation and activation. Moreover, it interferes with the expression of monocyte chemotactic protein (MCP)-1, TNF, interferoninducible protein-10 (IP-10), and IL-8. Due to the significant effect on pro-inflammatory cytokine signalling and impairment of adaptive immunity, there is concern that ustekinumab may increase the risk for infections, but to date, the available data is limited to case reports (47) and current studies have not demonstrated a significantly increased risk of pneumonia. However, the incidence of nasopharyngeal infections is increased by up to $10 \%$ (48). As many trials of ustekinumab were in individuals previously screened for latent $M$. tuberculosis infection, there is no demonstrable increased risk of tuberculosis reactivation, and the manufacturer continues to recommend that patients are screened for latent $M$, tuberculosis infection before initiation of treatment (49).

\section{Biological therapy in cancer}

Biological therapies, also known in this context as immunotherapies, have emerged as game changers in the treatment of various types of cancer (e.g., haematological, skin, gastric, breast, renal, urothelial and lung malignancies). Although many of these drugs are new, others have long been established in the treatment of cancer; For example, the FDA approved Bacillus Calmette-Guérin (BCG) in 1990 for the intravesical treatment of bladder cancer. These therapies mainly aim to alter the host's immune response so that it can detect and eliminate the cancer cells or inhibit tumour growth. Immunotherapies largely consist of (I) monoclonal antibodies that target antigens on the cell surface enabling the immune system to destroy them (e.g., alemtuzumab); (II) monoclonal antibodies that that inhibit checkpoint molecules to prevent cancer cells from evading the immune system (e.g., nivolumab); (III) monoclonal antibodies that inhibit cancer growth factors (e.g., bevacizumab); (IV) immunoconjugates containing antibodies that target specific antigens and deliver cytotoxic or radioactive substances (e.g., ado-trastuzumab emtansine); (V) cytokines that stimulate the immune system and induce cell apoptosis (e.g., interferons); and (VI) cancer vaccines that contain cancer-associated antigens to stimulate T-cells to kill cancer cells (e.g., sipuleucel-T).

The most notable adverse effects from immunotherapy are due to stimulation of the immune response, and these may mimic infection by causing flu-like symptoms and breathlessness due to pneumonitis. In a study of patients receiving checkpoint inhibitors, the incidence of pneumonitis was $5-10 \%$ with the highest frequency seen in those receiving combination treatment with antiPD1 and anti-CTLA4 inhibitors (50). In these cases, infectious aetiologies need to be excluded before initiation of treatment. The management of immune-related adverse events is with immunosuppressants (e.g., high dose corticosteroids, mycophenolate mofetil or TNF inhibitors) and these drugs will increase the risk of subsequent respiratory infection in particular with pathogens such as $M$. tuberculosis, $P$. jirovecii, A. fumigatus and the herpes viruses $(2,4,51,52)$. In a study of 740 patients receiving checkpoint inhibitors for the treatment of melanoma bacterial pneumonia, $P$. jirovecii pneumonia and invasive pulmonary aspergillosis were identified in $1.8 \%, 0.4 \%$ and $0.3 \%$ of patients respectively (53), suggesting that although rare, a high index of suspicion is required to identify these culprit pathogens. Like the PD-1 inhibitors, the PD-L1 inhibitors 
(e.g., atezolizumab, avelumab) are also associated with pneumonitis and development of severe respiratory infection in up to $5 \%$ of patients $(54,55)$. Other commonly used biologicals that are reported to increase the risk of respiratory infections include the monoclonal antibodies targeting CD20 (e.g., rituximab, ofatumumab, obinutuzumab, tositumomab, ibritumomab tiuxetan), that lead to B-cell depletion and toxicity causing neutropenia (56-58), monoclonal antibodies targeting CD52 (e.g., alemtuzumab) that cause B-cell lysis, T-cell depletion and neutropenia (59), and monoclonal antibodies targeting CD33 (e.g., gemtuzumab) that results in myelosuppression (60). Use of the cytokine IL-2 (aldesleukin) is also associated with an increased risk of respiratory infections due to neutrophil dysfunction (61). Importantly, the risk of respiratory infections with biological agents in the treatment of cancer is further augmented by their use in combination with immunosuppressive chemotherapeutic agents that frequently result in cytopenia.

Most of these infections can be prevented with prophylactic medication, but the frequency currently remains too low to justify their routine use and further research using larger cohorts are needed to demonstrate their cost-effectiveness. It is also important to note that discontinuation of the biological agent may not lead to rapid cessation of immunosuppression due to the long half-life of most monoclonal antibodies. Furthermore, in the presence of active infection discontinuation of immunosuppressive agents may lead to paradoxical worsening as the recovering immune system reacts to the pathogen, as seen in immune reconstitution inflammatory syndrome seen in the treatment of human immunodeficiency virus infection.

\section{Conclusions}

Biological therapies represent a revolution in the treatment of chronic inflammatory diseases and cancer, especially in cases refractory to conventional treatment modalities. They offer significant benefits in disease control and improving quality of life. As with all immunosuppressant agents, it is important to be aware and vigilant for signs of infection, as these therapies confer a significantly increased risk of respiratory infections.

\section{Acknowledgements}

None.

\section{Footnote}

Conflicts of Interest: The authors have no conflicts of interest to declare.

\section{References}

1. Doran MF, Crowson CS, Pond GR, et al. Frequency of infection in patients with rheumatoid arthritis compared with controls: A population-based study. Arthritis Rheum 2002;46:2287-93.

2. José RJ, Brown JS. Opportunistic bacterial, viral and fungal infections of the lung. Medicine (Baltimore) 2016;44:378-83.

3. Ali T, Kaitha S, Mahmood S, et al. Clinical use of antiTNF therapy and increased risk of infections. Drug Healthc Patient Saf 2013;5:79-99.

4. Jose RJ, Dickey BF, Brown JS. Infectious respiratory disease in non-HIV immunocompromised patients. Br J Hosp Med (Lond) 2014;75:685-90.

5. Hastings R, Ding T, Butt $S$, et al. Neutropenia in patients receiving anti-tumor necrosis factor therapy. Arthritis Care Res (Hoboken) 2010;62:764-9.

6. Singh JA, Wells GA, Christensen R, et al. Adverse effects of biologics: a network meta-analysis and Cochrane overview. Cochrane Database Syst Rev 2011;(2):CD008794.

7. Singh JA, Cameron C, Noorbaloochi S, et al. Risk of serious infection in biological treatment of patients with rheumatoid arthritis: a systematic review and metaanalysis. Lancet 2015;386:258-65.

8. Strangfeld A, Eveslage M, Schneider M, et al. Treatment benefit or survival of the fittest: what drives the timedependent decrease in serious infection rates under TNF inhibition and what does this imply for the individual patient? Ann Rheum Dis 2011;70:1914-20.

9. Salvana EM, Salata RA. Infectious complications associated with monoclonal antibodies and related small molecules. Clin Microbiol Rev 2009;22:274-90, Table of Contents.

10. Galloway JB, Hyrich KL, Mercer LK, et al. Anti-TNF therapy is associated with an increased risk of serious infections in patients with rheumatoid arthritis especially in the first 6 months of treatment: updated results from the British Society for Rheumatology Biologics Register with special emphasis on risks in the elderly. Rheumatology 2011;50:124-31.

11. Maini R, St Clair EW, Breedveld F, et al. Infliximab 
(chimeric anti-tumour necrosis factor alpha monoclonal antibody) versus placebo in rheumatoid arthritis patients receiving concomitant methotrexate: a randomised phase III trial. ATTRACT Study Group. Lancet 1999;354:1932-9.

12. Lanternier F, Tubach F, Ravaud P, et al. Incidence and Risk Factors of Legionella pneumophila Pneumonia During Anti-Tumor Necrosis Factor Therapy. Chest 2013;144:990-8.

13. Solovic I, Sester M, Gomez-Reino JJ, et al. The risk of tuberculosis related to tumour necrosis factor antagonist therapies: a TBNET consensus statement. Eur Respir J 2010;36:1185-206.

14. Winthrop KL, Yamashita S, Beekmann SE, et al. Mycobacterial and Other Serious Infections in Patients Receiving Anti-Tumor Necrosis Factor and Other Newly Approved Biologic Therapies: Case Finding through the Emerging Infections Network. Clin Infect Dis 2008;46:1738-40.

15. Saliu OY, Sofer C, Stein DS, et al. Tumor-Necrosis-Factor Blockers: Differential Effects on Mycobacterial Immunity. J Infect Dis 2006;194:486-92.

16. Tubach F, Salmon D, Ravaud P, et al. Risk of tuberculosis is higher with anti-tumor necrosis factor monoclonal antibody therapy than with soluble tumor necrosis factor receptor therapy: The three-year prospective French Research Axed on Tolerance of Biotherapies registry. Arthritis Rheum 2009;60:1884-94.

17. Lalvani A, Millington KA. Screening for tuberculosis infection prior to initiation of anti-TNF therapy. Autoimmun Rev 2008;8:147-52.

18. Lee JH, Slifman NR, Gershon SK, et al. Life-threatening histoplasmosis complicating immunotherapy with tumor necrosis factor alpha antagonists infliximab and etanercept. Arthritis Rheum 2002;46:2565-70.

19. Bergstrom L, Yocum DE, Ampel NM, et al. Increased risk of coccidioidomycosis in patients treated with tumor necrosis factor alpha antagonists. Arthritis Rheum 2004;50:1959-66.

20. Postmarket Drug Safety Information for Patients and Providers. Information for Healthcare Professionals: Cimzia (certolizumab pegol), Enbrel (etanercept), Humira (adalimumab), and Remicade (infliximab). Available online: https://www.fda.gov/drugs/drugsafety/postmarketdrugsafe tyinformationforpatientsandproviders/ucm124185.htm

21. Knox KS. Perspective on Coccidioidomycosis and Histoplasmosis. Am J Respir Crit Care Med
2014;189:752-3.

22. Wallis RS, Broder MS, Wong JY, et al. Granulomatous Infectious Diseases Associated with Tumor Necrosis Factor Antagonists. Clin Infect Dis 2004;38:1261-5.

23. Kaur N, Mahl TC. Pneumocystis jiroveci (carinii) Pneumonia After Infliximab Therapy: A Review of 84 Cases. Dig Dis Sci 2007;52:1481-4.

24. Harigai M, Koike R, Miyasaka N, Pneumocystis Pneumonia under Anti-Tumor Necrosis Factor Therapy (PAT) Study Group. Pneumocystis Pneumonia Associated with Infliximab in Japan. N Engl J Med 2007;357:1874-6.

25. Kim SY, Solomon DH. Tumor necrosis factor blockade and the risk of viral infection. Nat Rev Rheumatol 2010;6:165-74.

26. Murray E, Perry M. Off-label use of rituximab in systemic lupus erythematosus: a systematic review. Clin Rheumatol 2010;29:707-16.

27. Lu TY, Ng KP, Cambridge G, et al. A retrospective seven-year analysis of the use of $B$ cell depletion therapy in systemic lupus erythematosus at university college london hospital: The first fifty patients. Arthritis Rheum 2009;61:482-7.

28. Rosman Z, Shoenfeld Y, Zandman-Goddard G. Biologic therapy for autoimmune diseases: an update. BMC Med 2013;11:88.

29. Keystone E, Fleischmann R, Emery P, et al. Safety and efficacy of additional courses of rituximab in patients with active rheumatoid arthritis: An open-label extension analysis. Arthritis Rheum 2007;56:3896-908.

30. van Vollenhoven RF, Emery P, Bingham CO 3rd, et al. Longterm Safety of Patients Receiving Rituximab in Rheumatoid Arthritis Clinical Trials. J Rheumatol 2010;37:558-67.

31. Buch MH, Smolen JS, Betteridge N, et al. Updated consensus statement on the use of rituximab in patients with rheumatoid arthritis. Ann Rheum Dis 2011;70:909-20.

32. Jordan N, Lutalo PM, D'Cruz DP. Novel therapeutic agents in clinical development for systemic lupus erythematosus. BMC Med 2013;11:120.

33. Dennis GJ. Belimumab: A BLyS-Specific Inhibitor for the Treatment of Systemic Lupus Erythematosus. Clin Pharmacol Ther 2012;91:143-9.

34. Furie R, Petri M, Zamani O, et al. A phase III, randomized, placebo-controlled study of belimumab, a monoclonal antibody that inhibits B lymphocyte stimulator, in patients with systemic lupus erythematosus. Arthritis Rheum 2011;63:3918-30. 
35. Cartmell T, Poole S, Turnbull AV, et al. Circulating interleukin-6 mediates the febrile response to localised inflammation in rats. J Physiol 2000;526 Pt 3:653-61.

36. Furst DE, Keystone EC, So AK, et al. Updated consensus statement on biological agents for the treatment of rheumatic diseases, 2012. Ann Rheum Dis 2013;72 Suppl 2:ii2-34.

37. Navarro-Millán I, Singh JA, Curtis JR. Systematic Review of Tocilizumab for Rheumatoid Arthritis: A New Biologic Agent Targeting the Interleukin-6 Receptor. Clin Ther 2012;34:788-802.e3.

38. Hoi AY, Littlejohn GO. Abatacept in the treatment of lupus. Expert Opin Biol Ther 2012;12:1399-406.

39. Cohen S, Radominski SC, Gomez-Reino JJ, et al. Analysis of Infections and All-Cause Mortality in Phase II, Phase III, and Long-Term Extension Studies of Tofacitinib in Patients With Rheumatoid Arthritis. Arthritis Rheumatol 2014;66:2924-37.

40. Winthrop KL, Yamanaka H, Valdez H, et al. Herpes Zoster and Tofacitinib Therapy in Patients With Rheumatoid Arthritis. Arthritis Rheumatol 2014;66:2675-84.

41. Kamal A, Khamashta M. The efficacy of novel B cell biologics as the future of SLE treatment: A review. Autoimmun Rev 2014;13:1094-101.

42. Soliris $®$ (eculizumab) Concentrated solution for intravenous infusion. Highlights of prescribing information. Cited 2017 Jul 31. Available online: http:// soliris.net/resources/pdf/soliris_pi.pdf

43. Brown JS, Hussell T, Gilliland SM, et al. The classical pathway is the dominant complement pathway required for innate immunity to Streptococcus pneumoniae infection in mice. Proc Natl Acad Sci U S A 2002;99:16969-74.

44. Fleischmann RM, Schechtman J, Bennett R, et al. Anakinra, a recombinant human interleukin-1 receptor antagonist (r-metHuIL-1ra), in patients with rheumatoid arthritis: A large, international, multicenter, placebocontrolled trial. Arthritis Rheum 2003;48:927-34.

45. Genovese MC, Cohen S, Moreland L, et al. Combination therapy with etanercept and anakinra in the treatment of patients with rheumatoid arthritis who have been treated unsuccessfully with methotrexate. Arthritis Rheum 2004;50:1412-9.

46. Settas LD, Tsimirikas G, Vosvotekas G, et al. Reactivation of Pulmonary Tuberculosis in a Patient With Rheumatoid Arthritis During Treatment With IL-1 Receptor Antagonists (Anakinra). J Clin Rheumatol 2007;13:219-20.

47. Lynch M, Roche L, Horgan M, et al. Peritoneal tuberculosis in the setting of ustekinumab treatment for psoriasis. JAAD Case Rep 2017;3:230-2.

48. Papp KA, Langley RG, Lebwohl M, et al. Efficacy and safety of ustekinumab, a human interleukin-12/23 monoclonal antibody, in patients with psoriasis: $52-$ week results from a randomised, double-blind, placebocontrolled trial (PHOENIX 2). Lancet 2008;371:1675-84.

49. Papp KA, Griffiths CE, Gordon K, et al. Long-term safety of ustekinumab in patients with moderate-to-severe psoriasis: final results from 5 years of follow-up. Br J Dermatol 2013;168:844-54.

50. Naidoo J, Wang X, Woo KM, et al. Pneumonitis in Patients Treated With Anti-Programmed Death-1/ Programmed Death Ligand 1 Therapy. J Clin Oncol 2017;35:709-17.

51. Fujita K, Terashima T, Mio T. Anti-PD1 Antibody Treatment and the Development of Acute Pulmonary Tuberculosis. J Thorac Oncol 2016;11:2238-40.

52. Sepkowitz KA. Opportunistic Infections in Patients with and Patients without Acquired Immunodeficiency Syndrome. Clin Infect Dis 2002;34:1098-107.

53. Del Castillo M, Romero FA, Argüello E, et al. The Spectrum of Serious Infections Among Patients Receiving Immune Checkpoint Blockade for the Treatment of Melanoma. Clin Infect Dis 2016;63:1490-3.

54. Weinstock C, Khozin S, Suzman D, et al. U.S. Food and Drug Administration Approval Summary: Atezolizumab for Metastatic Non-Small Cell Lung Cancer. Clin Cancer Res 2017;23:4534-9.

55. Gulley JL, Rajan A, Spigel DR, et al. Avelumab for patients with previously treated metastatic or recurrent non-smallcell lung cancer (JAVELIN Solid Tumor): dose-expansion cohort of a multicentre, open-label, phase $1 \mathrm{~b}$ trial. Lancet Oncol 2017;18:599-610.

56. Voog E, Morschhauser F, Solal-Céligny P. Neutropenia in patients treated with rituximab. N Engl J Med 2003;348:2691-4; discussion 2691-4.

57. Dillman RO. Radioimmunotherapy of B-cell lymphoma with radiolabelled anti-CD20 monoclonal antibodies. Clin Exp Med 2006;6:1-12.

58. Horning SJ, Younes A, Jain V, et al. Efficacy and Safety of Tositumomab and Iodine-131 Tositumomab (Bexxar) in B-Cell Lymphoma, Progressive After Rituximab. J Clin Oncol 2005;23:712-9.

59. Osterborg A, Karlsson C, Lundin J, et al. Strategies in the Management of Alemtuzumab-Related Side Effects. Semin Oncol 2006;33:S29-35.

60. Larson RA, Sievers EL, Stadtmauer EA, et al. Final report of the efficacy and safety of gemtuzumab ozogamicin 
(Mylotarg) in patients with CD33-positive acute myeloid leukemia in first recurrence. Cancer 2005;104:1442-52. 61. Proleukin ${ }^{\circledR}$ (Aldesleukin). FDA label information.

doi: 10.21037/arh.2017.09.03

Cite this article as: José RJ, Mouyis M. Biological therapies in the treatment of inflammatory disease and cancer: impact on pulmonary infection. Ann Res Hosp 2017;1:40.
2012. Cited 2017 Jul 19. Available online: https:// www.accessdata.fda.gov/drugsatfda_docs/ label/2012/103293s5130lbl.pdf 